\title{
APROXIMACIÓN ÉTICA CIENTÍFICA AL DOBLE EFECTO O ÚNICO BIEN POSIBLE EN EL ABORTO TERAPÉUTICO
}

\author{
Carlos Y. Valenzuela ${ }^{1}$
}

Resumen: El estudio muestra como la doctrina o principio del doble efecto (DDE) de una acción con efecto secundario negativo no puede aplicarse a todos los casos de aborto terapéutico (AT). Con el análisis de las causas e indicación de AT se demuestra que solo algunos de estos corresponden a DDE. Cuando no es el feto el que lleva a la madre a tener un embarazo de alto riesgo con amenaza de dańo severo o muerte para ella, el caso puede adscribirse a la DDE; cuando es el feto directa o indirectamente el que causa a lleva a ese riesgo materno, el caso no se adscribe a la DDE. Si el feto es la causa de la muerte inminente de la madre, la remoción de la causa, que es la terapia adecuada, coincide con matarlo; entonces la acción buena (salvar a la madre tratándola causalmente) es la misma que la mala (matar al feto), situación que no puede asimilarse sea a la DDE o a la doctrina del mal menor. Más aún, decidir no interrumpir el embarazo produciría la muerte de la madre y el feto. El caso debería adscribirse al principio del único bien posible.

Palabras clave: aborto terapéutico, doble efecto, ética, ética científica, único bien posible

Scientific ethical approach to double effect or only possible good in therapeutic abortion

Abstract: This study shows the inapplicability of the doctrine of double effect (DDE) to all the cases of therapeutic abortion (TA). The causes of the maternal risk define cases that cannot be included in the DDE. When it is not the embryo or fetus that produces the mother pathology the case can be assigned to the DDE. When the embryo or fetus produces directly or indirectly the process that threatens the mother's life the case cannot be assigned to the DDE. If the fetus is the cause of the imminent mother's death, the removal of the cause, that is the proper therapy, coincides with killing him or her; then the good action (to save therapeutically the mother) is the same to the bad action (to kill the fetus) and this situation cannot be assimilated either to the DDE or to the doctrine of the lesser evil. Deciding not to kill the fetus will produce the death of the mother and the fetus. So this situation should be ascribed to the principle of the unique possible well.

Key words: therapeutic abortion, double effect, ethics, scientific ethics, unique possible good

\section{Aproximação ética científica ao duplo efeito ou único bem possível no aborto terapêutico}

Resumo: $\mathrm{O}$ estudo mostra como a doutrina ou princípio do duplo efeito (DDE) de uma ação com efeito secundário negativo não pode aplicar-se a todos os casos de aborto terapêutico (AT). Com a análise das causas e indicaçáo de AT se demonstra que só alguns destes correspondem a DDE. Quando náo é o feto que leva a mãe a ter uma gravidez de alto risco com ameaça de dano severo ou morte para ela, o caso pode circunscrever-se à DDE; quando é o feto direta ou indiretamente quem causa ou leva a esse risco materno, o caso não se circunscreve a DDE. Se o feto é a causa da morte iminente da mãe, a remoção da causa, que é a terapia adequada, coincide em matá-lo; então a ação boa (salvar a mãe tratando-a causalmente) é a mesma que a má (matar o feto), situaçáo que não pode assimilar-se à DDE ou à doutrina do mal menor. Mais ainda, decidir não interromper a gravidez produziria a morte da mãe e do feto. O caso deveria circunscrever-se ao princípio do único bem possível.

Palavras-chave: aborto terapêutico, duplo efeito, ética, ética científica, único bem possível

\footnotetext{
${ }^{1}$ Programa de Genética Humana, ICBM, Facultad de Medicina, Universidad de Chile, Chile Correspondencia: cvalenzu@med.uchile.cl
} 


\section{Introducción}

Si bien el principio o doctrina del doble efecto (DDE, usaremos aquí doctrina y no principio) tiene un desarrollo extenso desde los escolásticos, hay estudios recientes que analizan críticamente esas teorías escolásticas y toman más bien la posición de la filosofía analítica anglosajona o un análisis jurídico más exhaustivo(1-3). Nuestro análisis es más bien desde la ética científica (EC), fundada en el suceder universal y su conocimiento científico; en este caso, en el conocimiento de las situaciones en las que se toman las decisiones (la situación gineco-obstétrica y personas involucradas), de sus causas científicamente demostradas o demostrables, de las intenciones y de las creencias involucradas(4-6). En el presente trabajo nos interesa, más bien, su aplicación a la no imputabilidad de la muerte fetal al realizar un aborto ante la muerte inminente de la embarazada. La DDE propone que si una acción intencionada a realizar un "bien" tiene como efecto asociado un "mal", este no sería imputable al agente que realiza la acción, en condiciones bien acotadas. La DDE se ha aplicado a múltiples situaciones como aborto(7), vejez y muerte inducida $(8,9)$, producción de embriones humanos(10) y otras. En obstetricia la situación parece simple: un embarazo del que se sigue riesgo de muerte de la embarazada tiene como tratamiento la remoción de la causa de ese riesgo, que en este caso está en la placenta, en el útero o en el feto (o embrión) mismo, y si para salvar a la madre la remoción o término del embarazo, en una etapa de inviabilidad del feto ex útero, conducirá a la muerte de ese feto, esta muerte sería inimputable a esa acción. Pero, ¿es lo mismo si la causa de riesgo para la embarazada son los anexos o el útero o si es el feto mismo? Este es el dilema que presentaremos $\mathrm{y}$ trataremos de resolver.

En general la DDE acompaña a la ética desde un principio; toda acción humana direccionada a un fin nunca tiene una sola consecuencia (ese fin), sino que genera efectos variados o pleiotrópicos distintos al fin perseguido; la nomenclatura de doble efecto es didáctica y simplificadora $(2,3)$ y considera el efecto secundario mayor que nos preocupa, pero debería tratarse como la doctrina del efecto múltiple de toda acción(2-6). Toda decisión implica la omisión de otras alternativas a ella, y en sentido propio y específico omitir la no decisión específica, situación fundamental en EC, pero no crítica en otras éticas. Si tomo un vaso de agua omití el no tomar ese vaso de agua; decidir no tomar el vaso de agua tiene múltiples consecuencias con sus responsabilidades anexas. En relación a las decisiones y acciones hay un debate de varios milenios entre la aproximación de Heráclito — seguida por Hegel — y la de Aristóteles — seguida por Tomás de Aquino- - para quienes es válida la separación en potencia y acto. La DDE como la plantea el Aquinatense ha sido examinada críticamente en su uso legal $(2,3)$. Para el Estagirita y el Aquinatense las acciones se asocian a responsabilidades directas en relación a las intenciones y fines buscados, y sus consecuencias distintas de este fin pueden manejarse de acuerdo a este principio del doble o múltiple efecto $(2,3,11)$.

\section{Desarrollo}

\section{Brevia sobre ética cientifica}

En el presente análisis aplicaremos la ética científica(4-6) más acorde con la dialéctica heraclitiana, no de acto y potencia sino de un continuo devenir dinámico como estructura del ser: se es responsable en toda acción que se realiza tanto por lo que se hace como por lo que se deja de hacer y esto es inexorable, dado que - la EC muestra- no se puede no decidir. La EC parte del entendimiento de toda situación a analizar estudiada desde las ciencias, las percepciones, las concepciones y valoraciones comunes. La EC se adscribe a la matriz disciplinar de la decisión como elemento-unidad fundamental de la ética, entendida como la teoría y praxis del bien y del mal. Toda decisión implica un movimiento hacia alguna parte, de allí que la EC es sentido o significado motor(4-6). Las decisiones son entendidas en todo ser o proceso, por lo que la EC no es antropocéntrica: existe en todo el cosmos. Con todo, la decisión humana es estudiada por las neuro-psico-socio-ciencias y este estudio es basal para la EC, incluso se está desarrollando la neuroética(12), fundada principalmente en las neurociencias, bajo el postulado de la moral como materio-energeticidad de la arquitectura y función de la circuitería cerebral. La EC es mucho más amplia y considera a las neurociencias como uno de los elementos a ser tomado en cuenta, pero también considera a la evolución cósmica y orgánica, a la genética del comportamiento, a la antropología 
cultural, a las religiones, ideologías y creencias, etc. Así, el primer principio de la EC es la segunda ley de la termodinámica, que implica un movimiento desde un orden no azaroso hacia un orden azaroso (en epistemología científica y en EC el azar es un orden admirable). Su segundo principio es el de historicidad o evolución cósmica, orgánica y del ser humano(4-6). Para la EC es el proceso evolutivo el que produjo al ser humano y a su pensar, sentir o vivir, incluida su ética. No es el pensar del ser humano el que produjo la evolución. ¿Cuál era la ética que tenía Neanderthal u Homo erectus o un dinosaurio? En esta perspectiva de EC, que entiende el bien y mal óntico (como presencia o ausencia cósmica) independiente del ser humano y al bien convencional o gnósico como plural entre las culturas, la bondad o maldad tanto de la acción (hacer, cometer) como de la no acción (no hacer, omitir) tienen una catalogación valórica solo al interior de esas culturas, ideologías o religiones(4-6). La EC pretende no tan solo ser una disciplina teórico-práctica para iniciados o especialistas sino que para la persona común que puede encontrar en ella, dada la aceptación de la sociología y antropología cultural, los conocimientos, posiciones, factores emotivos, afectivos, valóricos, ideológicos o religiosos desde los que decidir en cualquier situación de la vida cotidiana o especializada, como es el caso que aquí estudiamos.

Desde la EC y en sentido legal o moral, los efectos de los actos, sean por sus consecuencias buscadas (intencionadas) o secundarias (no intencionadas pero, muy importante, conocidas), se pueden catalogar (en una primera aproximación) como laudables (buenos) o culpables (malos o dolosos), y según algunos como neutros(2,3). Las consecuencias desconocidas de las acciones que la ciencia y la experiencia humana irán encontrando o no encontrarán nunca no entran en el análisis o imputación en el tratado ético o bioético tradicionales, pero desde la EC nos obligan a ser humildes y modestos en la pretensión de bondad o maldad de nuestras obras y limitarnos a una sinceridad radical de intenciones y conocimiento. Científicamente se complica el análisis, dado que, según la teoría del caos determinista $(13,14)$ o de sistemas complejos, una acción al parecer insignificante puede tener un efecto catastrófico a largo plazo (efecto mariposa(15)). La administración masiva de un anticonceptivo puede solucionar un problema familiar-demográfico o social por un cierto tiempo, pero puede llevar a la extinción de la especie Homo sapiens. ¿Cuál es el juicio de bondad o maldad de ambos efectos (anticoncepción y extinción de la especie) por el que se condena o se absuelve, o se aplica esta droga? Por otra parte, la aplicación de las ecuaciones de onda de la física cuántica puede plantear historias paralelas, como el conocido caso del gato de Shrödinger(16), en el que se superponen probabilísticamente la posibilidad del gato de estar vivo y muerto. El tratamiento clásico ha sido que hay un solo sentido bueno-malo en esta catalogación y las dificultades se solucionan por la "razón". El tratamiento en EC es que hay tantos sentidos de bondad-maldad (gnósica) como religiones o ideologías se pronuncien al respecto; para la EC la razón no es la herramienta para catalogar como bueno o malo el efecto de una acción o ella misma (o su no acción), sino que hay creencias o suposiciones radicales al respecto, que no provienen de la razón sino de la creencia o convicción sobre la bondad o maldad de esas acciones, o que surgen primariamente desde "adentro", del fondo de sabiduría o creencias de ese ser humano(17). Por ejemplo, aunque no es homologable totalmente a la DDE, en una disyuntiva de dos caminos (estudios muy cruciales en neuroética, pero no tanto en EC), si tomando uno se mata a una mujer embarazada y tomando el otro se mata a una cucaracha con sus crías, en occidente nadie dudaría en tomar el segundo camino, pero no sería tan fácil para un hinduista o budista, para quienes los animales, aun las cucarachas, son también personas. Podemos homologarlo más bien con la muerte del parásito intestinal por ingestión de una droga para matarlo; lo que se quiere primariamente es curar al paciente con parasitosis pero secundariamente se mata a un animal, que es en esas culturas persona.

El análisis se complica si consideramos, más que bondad o maldad radicalmente opuestas, el posible problema de la existencia de grados de bondad o maldad o, como sucede en el hinduismo y budismo, en los que no hay tal diferencia absoluta, con un avance inexorable hacia la fusión (bondad) con la divinidad que constituye el bien eterno definitivo. No es distinta a la teoría de la apocatástasis (restauración total y definitiva) del cristianismo por la que aun el demonio al fin se salva $(18,19)$ y el infierno es evacuado totalmente. 
Tenemos aquí una diferencia más grande entre la EC y la neuroética(12) de naturaleza metodológica: aunque ambas usan la neurociencia en sentido experimental, la EC da mucha más importancia a la ciencia observacional de fenómenos más globales y evolutivos y de una crítica más amplia a las formas epistemológicas que subyacen en sus supuestos. No podemos entrar en detalle, pero para la EC poner a una persona frente a una situación imaginaria construida por el investigador puede informar algo sobre procesos "éticos" involucrados, pero no debe olvidarse que es un constructo artificioso que posiblemente nunca se de igualmente en la vida "natural", y que esa diferencia sea capital para constituirse en una acción ética. Pensar o razonar sobre algo es siempre reduccionista para la EC y los constructos experimentales no escapan a esta condición. Por otra parte, las neurociencias de las creencias profundas y de los estados paranormales evidenciados objetivamente están recién desarrollándose.

Se tiende a catalogar (acción de enjuiciar o etiquetar, impropia en EC) la posición de hacer depender la bondad o maldad de los actos de la ideología o religión de la persona de "ética relativista". Este es un error. Si para un hinduista criar animales, matarlos y comérselos es un acto "malo", y para un cristiano las mismas acciones son "buenas" y se hacen, no es posible unir estas posiciones; discrepan radicalmente, y no es relativismo que existan las dos; podrá haber confrontación, guerra entre ellas o tolerancia, respeto o amor, pero el relativismo allí es no aplicable. El relativismo existe al interior de una ideología o religión cuando un principio o fundamento no es válido en ciertas situaciones o condiciones, es decir, generalizando, que todo (toda bondad o maldad) principio es dependiente de las situaciones donde se aplica. Si hay bondades o maldades contradictorias entre religiones o ideologías, una solución sería buscar el bien óntico suprarreligioso o supraideológico; pero me temo que estamos muy lejos de tener una actitud en el ámbito mundial para hacerlo o que tal proyecto sea imposible.

\section{La DDE en el aborto}

Examinemos la DDE aplicada al aborto. Consolidando varias exposiciones, la DDE condiciona la no imputabilidad de un efecto secundario de una acción si (1-3,11): i) la acción, el objetivo (o más el efecto buscado con ella) y la intención con que se hace deben ser buenos, no malos o neutros (indiferentes); ii) la acción directamente no intenta mal alguno; iii) el efecto bueno no es consecuencia del mal, este no es un medio para conseguir un bien; iv) el resultado bueno debe ser mayor (mejor) que el efecto malo (proporcionalidad). Desde la EC vemos que estas consideraciones sí son relativas a las ideologías o visiones de mundo, ya que bueno, malo o mejor no tienen significado areligioso o anideológico para muchas situaciones. En la práctica resulta a veces imposible disociar los efectos, porque la situación de base del bien que se quiere buscar está entremezclada con el mal que se produce; disociar las acciones de sus efectos múltiples o pleiotrópicos no deja de ser arbitrario. Así, no continuaremos con este análisis general y estudiaremos la situación específica.

"Aborto provocado" ha sido definido como la interrupción voluntaria e intencionada del embarazo con feto inviable ex útero. La edad fetal para su viabilidad ex útero ha ido disminuyendo y se acepta ahora 20 semanas o peso inferior a 400 gramos(20). Para que haya aborto, tanto ética particularmente en EC - como legalmente, debe darse tres condiciones: i) el feto muera in útero; ii) la intención directa sea matarlo; iii) el feto debe ser humano. Nos estamos refiriendo al aborto provocado ético o legal, es decir, con responsabilidad moral o jurídica. De allí que el aborto de una mola hidatidiforme no es aborto ya que la mola no se considera humana(21); igualmente se ha propuesto que al triploide humano no se le considere humano y la interrupción del embarazo en este caso no sería aborto(21), situación propuesta para los anencéfalos o acráneos(22), lo que es motivo de discrepancia(23).

\section{Caso de aplicación correcta de la DDE}

El caso más común del "aborto terapéutico" en el que la DDE se aplica es el aborto séptico. La embarazada ha tratado de abortar introduciéndose algún elemento por el cuello uterino, no se ha producido el aborto pero se ha infectado el huevo con sepsis secundaria, estado febril sobre $40^{\circ} \mathrm{C}$ e inminencia de muerte de la embarazada por shock séptico. Se procede al vaciamiento uterino para terminar con el foco séptico, única forma de salvar a la madre; 
en general, el feto o embrión ya está muerto o ha iniciado el proceso irreversible hacia la muerte. El caso puede ser homologado al de cáncer uterino en el embarazo o disposición o tipo de placenta incompatible con el progreso del embarazo. También puede asimilarse (no muy homologable) a este caso el embarazo ectópico, que de no intervenirse a tiempo puede comprometer gravemente la vida de la embarazada. Aquí la intención primaria es salvar a la madre removiendo la causa (que no es el feto mismo, excepto en el embarazo tubario, en el que el embrión origina y mantiene al trofoblasto que, a su vez, mata a la embarazada, por eso no es tan homologable) de su riesgo vital grave y no matar al feto como primera intención, por lo que se ajustaría a los criterios para considerar la acción bajo la DDE.

\section{Caso en que no se aplica la DDE}

En el caso en que el feto produce sustancias que directa o indirectamente matan a la madre o comprometen severamente su salud, o bien que el feto, por sus requerimientos metabólicos, lleva a una descompensación inmanejable de la homeostasis materna, es el feto, sus sustancias y su metabolismo la causa de la muerte o daño severo de la madre y solo su remoción puede salvarla. Este caso no es asimilable a la DDE, ya que la lex artis indica que, sabiendo la causa de la patología, si esta es removible debe ser removida, pero en este caso coincide con matar al feto. La decisión alternativa (y contradictoria) es no matar al feto y de ello se sigue la muerte de la madre con la consiguiente muerte del feto. Luego, o el médico mata al feto para salvar a la madre o no mata al feto y se mueren los dos. No es asimilable a la DDE porque: i) el efecto primario bueno que se busca es remover la causa de muerte de la embarazada que coincide con la vida y desarrollo del feto; luego coinciden, "en la acción", el efecto primario "bueno" y el secundario "malo" o, si se quiere, son la misma cosa (no es así en el ser mismo de la medicina como veremos más adelante); ii) el resultado alternativo a la comisión del acto (dialécticamente la no acción) es la omisión del acto que lleva al mismo resultado (que la comisión), al menos en relación a la vida del feto. Pero si matando al feto se logra al menos salvar a la madre, que es lo único posible, a esta conducta no la podemos asimilar a la DDE, pero tampoco completamente a la doctrina del mal menor, por- que salvar a la madre no es un mal, y solo parece serlo al considerar la hipotética pero no posible (con el conocimiento actual) alternativa de salvar a los dos; decir que salvar a la madre es un mal es un disfemismo (en sentido etimológico y no coloquial), infamia o blasfemia (se nos quedó corto el vocabulario porque ninguna calza bien). Entonces, al menos nominalmente, la única doctrina satisfactoria para esta decisión es la doctrina del único bien posible.

Los obstetras a veces cuestionan este análisis planteando que, de todos modos, no es la muerte del feto lo que se quiere y, por lo tanto, "aborto terapéutico” no podría ser aplicado a este caso. Puede demostrarse que, en el conocimiento actual de la medicina, "aborto terapéutico" es correcto, pues no queda otra alternativa que matar al feto (remoción de la causa de patología y por ende terapéutica) para salvar a la madre. La demostración proviene del caso de embarazo múltiple, en el que uno o más fetos, pero no todos, están causando el deterioro y muerte materna; en estos casos se ha matado selectivamente a ese o más fetos con lo que se ha conseguido salvar a la madre y a los otros fetos, llegando finalmente a un parto múltiple con uno o más fetos muertos y retenidos(24-26). Nótese que aquí no se produce la interrupción del embarazo ni tampoco el aborto entendido como expulsión ex útero de ese feto.

Esto nos permite aclarar que, en los argumentos de los legisladores y en el análisis ético, se comete un error grave al hacer sinónimos "aborto", "interrupción de embarazo" o "asesinato del feto" (o embrión). Son cosas muy distintas. Insistamos: el aborto legal o ético del que estamos tratando es la muerte intencionada del feto, se produzca o no la interrupción del embarazo o el aborto del feto. Matar intencionadamente a un feto in útero es un aborto legal o moral aunque no se interrumpa el embarazo. Por otra parte si se realiza la interrupción del embarazo y aborto (extracción del feto desde el útero) y se obtiene un feto vivo, este pasa a ser un recién nacido inmaduro, y su ética y legislación es muy diferente, ya que matarlo pasaría a ser un homicidio (infanticidio). Este es un problema gravísimo, no resuelto en el aborto de un feto malformado o disgenético, por lo que se ha planteado matarlo antes de extraerlo. 
Cabe considerar que dejar progresar el embarazo no siempre necesariamente implica la muerte de la madre, pero puede seguirse un daño severo, como quedar descerebrados ella o el feto u otras consecuencias. El análisis ético de cada una de estas posibilidades queda fuera del alcance de nuestro trabajo actual, podemos recomendar, en general, dejar la evaluación de la situación al equipo de salud y a la embarazada misma que, en su autonomía y considerando las posibilidades y riesgos que el equipo de salud le presenta, tome la decisión que en conciencia estime satisfactoria para ella. En estas situaciones, el acompañamiento del equipo de salud y profesionales adjuntos, más el del comité de ética asistencial pueden constituir, con la embarazada, una instancia muy integradora y la única satisfactoria para la decisión.

Hasta este punto, hemos hecho un análisis ajustado a los procesos directos. Un análisis más profundo, filosófico, científico o de teología moral escapa a este artículo. Recordemos la teoría de caos o el gato de Shrödinger ya mencionados. Solo recordaremos que en ese análisis más profundo se hace distinciones de la gravedad ética de las acciones de matar por acción o comisión, y de matar por omisión o dejar morir. Puede plantearse que no es homologable el matar directamente al feto (comisión) con el matarlo al no interrumpir (omisión) el embarazo con la consiguiente muerte de la embarazada y del feto. La primera es muerte por comisión y la segunda por omisión (pero pasa por la no despreciable acción de dejar morir a la madre pudiendo salvarla). Esto, que podría ser válido en un ambiente no médico, no lo es aquí, ya que si un médico no aplica la acción terapéutica que salva a su paciente de la muerte se hace responsable de su muerte. Más distinto se hace con el caso de la no homologación de la muerte por acción u omisión, al considerar que la omisión en este caso termina con la misma muerte del feto, que era lo que se pretendía evitar. El médico no tiene grados de libertad: mata al feto por comisión u omisión de esa acción. Otras comparaciones, como matar al feto versus no hacerlo y dejar a la madre descerebrada, quedan fuera del análisis por lo ya señalado.

Las implicancias de este análisis son numerosas pero solo indicaremos dos. En obstetricia se insiste que el aborto terapéutico no existe, porque nunca la intención primaria es matar al feto sino que sal- var a la madre, y se sigue el doble efecto negativo de la muerte del feto; esta posición no es sostenible, según nuestro análisis, porque en los casos en los que el feto directamente está matando a la madre y él es la causa de esa muerte, la remoción de la causa es matar al feto y por lo tanto es una muerte terapéutica del feto para, por lo menos, salvar a la madre, ya que de otra forma se la mata y con ello también se mata al feto por omisión. La segunda implicancia relacionada es que aparecen a lo menos dos situaciones bien distintas en la decisión de suspender el embarazo: una en que no es el feto directamente el que amenaza a la madre y otra en que sí lo es; ambas no pueden ser tratadas con el mismo predicamento o en el mismo paradigma ético.

La medicina (pero no el médico en esa contingencia) nunca tiene la intención de matar seres humanos (feto en este caso), ese es uno de sus principios centrales; matar a un ser humano feto o más desarrollado es un acto no médico y es incompatible con la intencionalidad nuclear de la medicina. El feto está matando a la madre por sustancias que emite o por competencia con su fisiología; si se tuviera la posibilidad de neutralizar esas sustancias con feto vivo o si se tuviera la terapéutica para suplir la deficiencia funcional materna esta sería la opción médica obligatoria. Por eso hemos dejado la puerta abierta de la distinción de matar "intencionadamente" al feto; esto no es así y solo ocurre porque no puede separarse la causalidad factual del agente matricida (sustancias que emite el feto) con el agente mismo (el feto). La medicina siempre ha tenido la intención de salvar a los dos; el progreso médico así lo demuestra, como es la posibilidad actual de ser viable ex útero para un feto de 20 semanas, lo que era impensable hace 30 años.

El caso de matar al feto antes que mate a la madre se asimila al caso de un demente que se sube a una torre con un rifle a matar personas: no se puede discutir si se lo neutraliza o si se lo mata cuando continúa disparando(21). La causa de muerte de esos transeúntes es el demente que emite balas que los matan, homologable en nuestro caso al feto que emite sustancias que matan a la madre. La solución y análisis son también homologables(21). 


\section{Referencias}

1. Stanford Encyclopedia of Philosophy. Doctrine of Double Effect. 2014. Disponible en http://plato.stanford.edu/entries/ double-effect/

2. Miranda AM. El principio del doble efecto y su relevancia en el razonamiento jurídico. Revista Chilena de Derecho 2008; 35(3): 485-519.

3. Araya PF, Navarro FI. El principio del doble efecto. Memoria para optar al grado de Licenciatura en Ciencias Jurídicas y Sociales. Santiago de Chile: Facultad de Derecho, Universidad de Chile; 2014.

4. Valenzuela CY. Ética científica y embriones congelados Revista Médica de Chile 2001; 129: 561-568.

5. Valenzuela CY. The Ontogenetic Origin of Human Beings in the Scientific-Ethics Perspective and its Implications on Abortion. Clinical Research and Bioethics 2013; 4(2): 148-154. doi 10.4172/2155-9627.1000148.

6. Valenzuela CY. Verso un'etica scientifica: l'origine della individualità ed il problema degli embrioni congelati. In: Redi CA, Caragna S, Zuccotti M, eds. Biologia delle cellule staminali. Opportunità e limiti di impiego. Pavía: Colegio Gisleri; $2000: 33-45$.

7. Prusak BG. Double effect, all over again: The case of Sister Margaret McBride. Theoretical Medicine and Bioethics 2011; 32: 271-283.

8. Capitaine L, Devolder K, Pennings G. Lifespan extension and the doctrine of double effect. Theoretical Medicine and Bioethics 2013; 34: 207-226.

9. Lindblad A, Lynöe, Juth N. End-of-life decisions and the reinvented rule of double effect: a critical analysis. Bioethics 2014; 28(7): 368-377.

10. Di Nucci E. Embryo loss and double effect. Journal of Medical Ethics 2013; 39: 537-540.

11. Kraus A. Principio del doble efecto. La Jornada-Unam. Disponible en: http://www.jornada.unam.mx/2005/07/27/index.php?section $=$ politica\&article $=022 \mathrm{a} 2 \mathrm{pol}$

12. Figueroa G. Las ambiciones de la neuroética: fundar científicamente la moral. Acta Bioethica 2013; 19(2): 259-268.

13. Ruelle D. Azar y Caos. Madrid: Alianza Universidad; 1991.

14. Enciclopedia Wikipedia. Teoría del Caos. Disponible en: https://es.wikipedia.org/wiki/Teor\%C3\%ADa_del_caos

15. Enciclopedia Wikipedia. Efecto Mariposa. Disponible en: https://es.wikipedia.org/wiki/Efecto_mariposa

16. EnciclopediaWikipedia. Gato deSchrödinger.Disponible en:https:/es.wikipedia.org/wiki/Gato_de_Schr\%C3\%B6dinger

17. Varela F. Ética y Acción. Santiago de Chile: Dolmen; 1996.

18. Quevedo AM. ¿Y si el diablo se salva? El debate por la eternidad del infierno y el seguimiento de Cristo. Reflexión del curso de Síntesis Teológica. Reflexiones Teológicas (Bogotá, Colombia) 2013; 11: 63-77.

19. Enciclopedia Wikipedia. Apocatástasis. Disponible en: https://es.wikipedia.org/wiki/Apocat\%C3\%A1stasis

20. Bórquez G, Bernier L, Besio MR, Montt JM, Novoa FS, Salas SI, et al. Reflexiones en torno al proyecto de ley (boletín $\mathrm{n}^{\circ}$ 9895-11) que regula la despenalización de la interrupción voluntaria del embarazo en tres causales. Revista Chilena de Obstetricia y Ginecología 2015; 80(3): 199-207.

21. Valenzuela CY. Ética científica del aborto terapéutico. Revista Médica de Chile 2003; 131(5): 562-568.

22. Valenzuela CY. Ética científica del aborto en el caso de anencefalia. Revista Médica de Chile 2011; 139: 1235-1239.

23. Besio M, Besio FH. Estatuto ontológico y ético del feto anencefálico: una perspectiva filosófica. Revista Médica de Chile 2008; 136: 783-788.

24. Heyborne KD, Porreco RP. Selective fetocide reverses preeclampsia in discordant twins. American Journal of Obstetrics \& Gynecology 2004; 191: 477-480.

25. Lu J, Ting YH, Law KM, Lau TK, Leung TY. Radiofrequency Ablation for Selective Reduction in Complicated Monochorionic Multiple Pregnancies. Fetal Diagnosis and Therapy 2013; 34: 211-216.

26. Arlicot IC, Potin J, Simon E, Perrotin F. Interruption sélective sur grossesse gémellaire monochoriale: étude nationale des pratiques des Centres pluridisciplinaires de Diagnostic Prénatal. Gynécologie Obstétrique et Fertilité 2014; 42: $387-392$.

Recibido : 10 de febrero de 2016

Aceptado : 17 de marzo de 2016 